\title{
La Ética Pública en México y su relación con los Derechos Humanos y el Derecho Penal
}

Public Ethics in Mexico and its relationship with Human Rights and Criminal Law

\section{(c) $(1) \Theta(0)$ \\ Esta obra está bajo licencia Creative Commons Attribution-NonCommercial-ShareAlike $\quad 4.0$ International (CC BY-NC-SA 4.0)}

Rodolfo Rafael Elizalde CASTAÑEdA EDgar RAMÓN AgUILERA GARCÍA ${ }^{2}$

\author{
$0^{\circ}$ IUS Comitiãlis / Año 4, Número 7 / enero - junio 2021 / pp. 192-216 / ISSN: 2594-1356
}

Recepción: 14 de noviembre de 2020 / Aceptación: 13 de marzo de 2021

Resumen: El objetivo de este artículo fue analizar comparativamente los códigos de ética para los servidores públicos federales que han estado vigentes en México y su relación con los derechos humanos y el derecho penal, con el fin, primero, de conocer la forma en que han evolucionado dichos códigos a partir de sus principios y valores éticos, segundo, estudiar el vínculo que guardan estos con las reformas constitucionales en derechos humanos de 2011 y en derecho penal del 18 de junio de 2008, para comprender el motivo por el que algunas organizaciones no gubernamentales (ONGs), como Human Rigth Watch (HRW), Transparencia Internacional (TI), Latinobarómetro, World Justice Proyect (WJP) y México Evalúa, siguen ubicando a México en los primeros lugares de corrupción e impunidad.

Palabras clave: Ética pública, organizaciones no gubernamentales, derechos humanos, corrupción, impunidad.

Abstract: The objective of this article was to comparatively analyze the codes of ethics for federal public servants that have been in force in Mexico and their relationship with human rights and criminal law, in order, first, to know how they have evolved these codes from their principles and ethical values, second, study the link that these have with the constitutional reforms in human rights of 2011 and in criminal law of June 18, 2008, to Right Watch (HRW), Transparencia Internacional (TI), Latinobarómetro, World Justice Project (WJP) and Mexico Evalúa, continue to place Mexico in the first places of corruption and impunity.

Key words: Public ethics, non-governmental organizations, human rights, corruption, impunity.

https://orcid.org/0000-0001-8680-3581. / Correo electrónico: rodolfoelizaldecas@yahoo.com.mx

${ }^{1}$ Facultad de Derecho, Universidad Autónoma del Estado de México, Toluca, Estado de México, México.

https://orcid.org/0000-0003-0119-0515. / Correo electrónico: edgaraguilera50@hotmail.com

${ }^{2}$ Facultad de Derecho, Universidad Autónoma del Estado de México, Toluca, Estado de México, México. 


\section{INTRODUCCIÓN}

El 31 de julio de 2002 fue publicado en el Diario Oficial de la Federación (DOF) el primer código ético de los servidores de la administración pública federal en México (CESAPF-2002), en abril de ese mismo año la Oficina del Alto Comisionado de las Naciones Unidas para los Derechos Humanos (OACNUDH) y el gobierno mexicano, firmaron un acuerdo que dio inició al Diagnóstico sobre la situación de los derechos humanos en México; es decir, casi al mismo tiempo que con este primer código de ética se establecen las reglas y principios del comportamiento de los servidores públicos federales, el principal organismo mundial responsable de los derechos humanos abre una investigación para conocer la situación de los derechos humanos en México. En el prólogo de esa investigación se dice de México:

Éste es uno de los pocos países del mundo en los que las Naciones Unidas han emprendido esa tarea, y es el primer caso en que la misma se realiza con la participación de representantes de organismos civiles y organismos gubernamentales, en el marco de un proceso abierto e independiente (2003, p. IV).

Dicha investigación se basó en una relación fáctica tendiente a revisar las causas estructurales de la violación a los derechos humanos en México y la identificación de algunas de sus principales deficiencias, esto es, corrupción e impunidad. Asimismo, establece que:

El Diagnóstico recoge demandas que constituyen un clamor de la sociedad mexicana, y que en la mayoría de los casos también están reflejadas en las recomendaciones y diversos informes que en materia de derechos humanos han realizado sobre el país la Organización de las Naciones Unidas y la Organización de Estados Americanos, por medio de sus distintos mecanismos de protección, así como la Comisión Nacional de los Derechos Humanos y diversas organizaciones no gubernamentales nacionales e internacionales (2003, p. V).

Ahora bien, de las 31 recomendaciones que se presentaron en el informe relacionado con el citado Diagnóstico, se observa al gobierno mexicano como principal violador de los derechos humanos, abarcando — hay que decirlo— todos los ámbitos de los poderes públicos (Ejecutivo, Legislativo y Judicial), los gobiernos federales, locales y organismos autónomos. Nada ni nadie escapó a la lupa de esa investigación, bastaría leer las recomendaciones citadas para darnos cuenta de que en 2003 los derechos humanos y el Estado de Derecho estaban prácticamente resquebrajados en México, pues la corrupción y la impunidad estaban por todos lados. Cabe mencionar que la metodología que se utilizó fue participativa habiendo consultores, asociaciones civiles, foros, trabajo de campo, seminarios, entrevistas con las víctimas, etc. El proble- 
ma que se plantea en este trabajo radica en que a diecisiete años de los resultados de ese Diagnóstico no solo ha habido cuatro códigos de ética para los servidores públicos federales, sino que, además, en el 2011 se publicó la reforma constitucional en Derechos Humanos, pero actualmente, los estudios de organizaciones no gubernamentales (ONGs), como Human Rigth Watch (HRW), Transparencia Internacional (TI), Latinobarómetro, World Justice Proyect (WJP), México Evalúa, ubican a México en los primeros lugares de corrupción e impunidad.

Por lo anterior, se considera que este ejercicio académico se justifica, pues los resultados que arroje servirán para buscar respuestas que ayuden a los estudiosos de los derechos humanos, la ética y, en general, a los interesados en la ciencia jurídica a contar con mayores argumentos que les permitan conocer de una manera más profunda, no solamente las causas y motivos de esas conclusiones, sino el vínculo que guardan los principios y valores contenidos en los mencionados códigos de ética con los derechos humanos, los resultados que arrojan los estudios que realizan las ONGs de referencia, en especial, sobre los temas de impunidad y corrupción. Pues, sin lugar a duda, hay un divorcio entre los citados avances legislativos con la realidad que arrojan los referidos estudios. Entre algunos antecedentes que encontramos sobre este tema, está una tesis titulada Importancia de la formación ética de los servidores públicos y de la aplicación de la ley para garantizar un desempeño íntegro (Hernández, et. al., 2017), pero que no se confronta con los resultados de las mencionadas ONGs ni con los cuatro códigos de ética, por lo que este estudio se aproxima, pero no es igual que el que se desarrolla en este ejercicio. Asimismo, el autor Óscar Diego especialista en dichos temas ha escrito el artículo titulado Los Códigos Éticos en el Marco de las Administraciones Públicas Contemporáneas. Valores para un Buen Gobierno (Diego, 2005) y el libro, Ética para corruptos: Una forma de prevenir la corrupción en los gobiernos y administraciones públicas (Diego, 2009). El primero, es un estudio sobre los Códigos de Ética en las administraciones públicas, es decir, es un estudio muy general, mientras que el segundo, es un estudio sobre la ética y la corrupción, enfocado al análisis sobre diferentes gobiernos y administraciones públicas del mundo y termina con la propuesta de un modelo ético integral para prevenir la corrupción, por lo que, ninguno de los dos productos se asemeja al trabajo que aquí se propone.

El presente estudio inicia a partir del año 2002, pues, por un lado, con el código de ética de ese año se fijaron las bases de actuación de la conducta de los servidores públicos federales, mientras que, en ese mismo año la ONU da inicio a la investigación que se apertura con motivo del citado Diagnóstico. El objetivo fue analizar comparativamente los códigos de ética para los servidores públicos federales que han estado vigentes en México y su relación con los derechos humanos y el derecho penal, con el fin, primero, de conocer la forma en que han evolucionado dichos códigos a partir de sus principios y valores éticos; segundo, estudiar el vínculo que guardan estos con las reformas constitucionales en derechos humanos de 2011 y en derecho penal del 18 de junio de 2008, para comprender el motivo por el que las organizaciones señaladas, no gubernamentales (ONGs), siguen ubicando a México en los primeros lugares de corrupción e impunidad. La base del estudio fue el método descriptivo, tomando en consideración el reducido espacio para publicar este tipo de trabajos. 
Las preguntas que orientaron este trabajo fueron, ¿Cuáles son las semejanzas y diferencias de los cuatro códigos de ética para los servidores públicos federales que han estado vigentes en México? ¿Cuál es el vínculo que guardan los principios y valores éticos de los cuatro códigos de ética que han estado vigentes en México, con los derechos humanos? ¿Cuáles son las causas por los cuales los estudios realizados por algunas ONGs (como HRW, TI, Latinobarómetro, WJP y México Evalúa) siguen ubicando a México en los primeros lugares de corrupción e impunidad? La hipótesis que formulamos supone que hay grandes semejanzas entre los principios y valores de los cuatro códigos de ética que han estado vigentes en México; sobre todo, hay, igualmente, lazos muy estrechos entre aquellos con los derechos humanos. Sin embargo, sigue habiendo una brecha muy grande entre todos estos con la realidad; por ese motivo, en los estudios realizados por las mencionadas ONGs sigue apareciendo México en los primeros lugares de corrupción e impunidad.

El marco teórico que da fundamento a este trabajo es la Teoría del Estado de Hermann Heller, la Teoría axiológica de Rudolf Smend, y la teoría del garantismo penal de Luigi Ferrajoli. La información utilizada proviene de la aplicación del método comparativo entre los cuatro Códigos de Ética vigentes en México, el análisis de las citadas reformas constitucionales, revisión de notas periodísticas, bibliografía especializada y los resultados de los estudios de las mencionadas ONGs.

\section{MARCO TEÓRICO CONCEPTUAL}

En este apartado nos referimos a algunas de las teorías y conceptos centrales que dan sustento a la investigación. Como señala Alessandro Pissoruzzo, "La existencia de múltiples puntos de contacto del Derecho Constitucional con otras muchas ciencias es algo admitido de modo generalizado." (Como se cita en Fernández, 1992, p. 1) El asunto que hoy nos ocupa, es uno de esos casos, pues es innegable que el Derecho Constitucional tiene una conexión muy estrecha con la ética, los derechos humanos y el derecho penal y, todos estos con la realidad, al estilo de Hermann Heller (Monereo, 2004, pp. 389-390), de quien destaca su punto de vista sobre lo teórico y lo práctico de la Teoría del Estado, refiriéndose a estudiar la realidad de la vida estatal y lo mismo afirmaba en relación con la Ciencia Política, por eso sostenía que esa relación entre ambas era indisoluble (Monereo, pp. 390-391). Siguiendo esta línea de pensamiento trazada por Heller, es que podemos decir que la ética, los derechos humanos y el derecho penal, además de la relación supra mencionada, tienen también la característica de lo teórico y lo práctico; para comprenderlas hay que confrontarlas con la realidad social. También, destaca en este ejercicio la teoría axiológica de los derechos fundamentales (Carbonell, 2004, pp. 40-41; Nogueira, 2003, p. 168), misma que se basa en la concepción del Estado como: "Una comunidad política en permanente proceso de integración en torno a unos valores, creencias y cultura. Los derechos y libertades se se (sic) reconocen como instrumentos a través de cuyo ejercicio se propicia ese proceso de integración." (Bastida, et. al., 2004, p. 62) Destaca de esta concepción, la relativización de esos derechos, pues su contenido depende de la interpretación que 
de los mismos valores se haga; además, corresponde al Estado establecer cuáles son dichos valores. Pero si tal decisión se toma con base en la mayoría, eso puede afectar los derechos de las minorías (Bastida, et al, 2004, p. 63). Sin embargo, esta teoría hace hincapié en que los valores tienen un sentido teleológico, de tal manera que si se presenta un conflicto entre ellos este se soluciona con la ponderación y es aquí donde se puede incurrir en un "subjetivismo y arbitrariedad" (Bastida, et al, 2004, p. 63). En relación con el derecho penal, tendremos presente a Luigi Ferrajoli con su obra, Derechos y garantías. La ley del más débil (2004), pues el garantismo constituye una de las teorías más influyentes, en especial, en ese ámbito del derecho mexicano. Basta citar su definición de derechos fundamentales: "todos aquellos derechos públicos subjetivos que corresponden universalmente a 'todos' los seres humanos en cuanto dotados del status de personas, de ciudadanos o de personas con capacidad de obrar" (Ferrajoli, 2004, p. 37). Una prueba de esa influencia se puede observar en el primer párrafo del artículo $1^{\circ}$ de la Constitución Política de los Estados Unidos Mexicanos (CPEUM), el cual señala que "En los Estados Unidos Mexicanos todas las personas gozarán de los derechos humanos reconocidos en esta Constitución y en los tratados internacionales de los que el Estado Mexicano sea parte ...”.

Por otra parte, podemos decir, parafraseando a Julius Stone, citado por George Nakhnikian (1993, 7-8) en su libro El derecho y las teorías éticas contemporáneas, que los códigos de ética contienen los ideales a los que se deben adecuar las conductas de los servidores públicos, lo que identifica como jurisprudencia ética. Pero, al mismo tiempo, Nakhnikian se cuestiona sobre cuáles son los "efectos reales del derecho sobre las actitudes y conductas de los hombres cuyo comportamiento regula...” (1993, pp. 7-8) lo que denomina jurisprudencia sociológica. También refiere que la ética contemporánea se divide en ética teórica y ética normativa. Asimismo, identifica a la jurisprudencia ética como ética normativa, toda vez que se ocupa de lo que debemos hacer o valorar; además, Nakhnikian (1993), agrega que "La ética normativa es aquella rama de la filosofía que trata de descubrir, justificar y formular principios normativos (reglas de conducta) ...”. O sea, conforme a estas ideas, la ética normativa está en consonancia con la ética teórica y viceversa.

\section{a. Ética.}

Jaqueline Prado Carrera (2016), refiere que la ética, "es la conducta del hombre frente a la responsabilidad que este tiene ante los ojos de otros hombres. Es decir, sobre lo que otros esperan de su actuación”. (p. 376). Por su parte, Yolimar Vilchez (2012) sostiene que, "La ética se refiere a la disciplina filosófica que constituye una reflexión sobre los problemas morales” (p. 234). Mientras que Julio De Zan (2004) señala que la ética es "la ciencia o disciplina filosófica que lleva a cabo el análisis del lenguaje moral y que ha elaborado diferentes teorías y maneras de justificar o de fundamentar y de revisar críticamente las pretensiones de validez de los enunciados morales" (p. 19).

\section{b. Corrupción.}

Irma Sandoval (2016, pp. 119-120) en su libro, Enfoque de la corrupción estructural: poder, impunidad y voz ciudadana, refiriéndose a la corrupción, cita a la Organización de las 
Naciones Unidas en el Programa Global contra la Corrupción, la cual define el fenómeno de la corrupción como un: "comportamiento de los individuos y funcionarios públicos que se desvían de las responsabilidades establecidas y usan su posición de poder para satisfacer fines privados y asegurar sus propias ganancias”. Por su parte, Rowland refiere que la corrupción es "el abuso de poder público para obtener beneficio particular". (2003, p. 3) Mientras que Roberto Ochoa (2017) sostiene que el término corrupción es entendido como, "cualquier actividad a través de la cual se altera y trastoca la forma y el objeto de una cosa, de un procedimiento o de una relación, a cambio de la promesa u obtención de beneficios recíprocos entre sus protagonistas". ${ }^{3}$ (p. 206). En síntesis, cuando nos referimos al ámbito público nos encontramos con aquellos que cometen sobornos, cohechos o malversaciones de fondos públicos; estos tuercen principios legales teniendo como resultado beneficios directos para los políticos o burócratas correspondientes.

\section{c. Impunidad.}

Según el Diccionario de la Lengua Española (2014), impunidad es una falta de castigo y etimológicamente significa "delito sin castigar". Coincidente con este significado, Juan Le Clercq, et. al. (2016) refieren que la impunidad hace referencia a un "acto de injusticia porque la persona o grupo de personas que cometieron el delito no fueron sancionadas o las víctimas no tuvieron el derecho a la reparación del daño” p. 72). Estos autores (2016) en su artículo "Midiendo la impunidad en América Latina: retos conceptuales y metodológicos”, se refieren a la definición de la Comisión de Derechos Humanos del Consejo Económico y Social de la Organización de las Naciones Unidas (ONU), que el 8 de febrero de 2005 presentó el Informe Orentlicher, en el cual se establece que:

Por impunidad se entiende la inexistencia, de hecho, o de derecho, de responsabilidad penal por parte de los autores de violaciones, así como de responsabilidad civil, administrativa o disciplinaria, porque escapan a toda investigación con miras a su inculpación, detención, procesamiento y, en caso de ser reconocidos culpables, condena a penas apropiadas, incluso a la indemnización del daño causado a sus víctimas (p. 6).

Sin embargo, Juan Le Clercq (2018) agrega que:

La impunidad no puede limitarse a la noción de crimen sin castigo o reducirse a un problema estrictamente legal, sino que se relaciona con climas sociales, ambientes políticos y la conducta de actores institucionales que permiten que los delitos, la ilegalidad y la violación de los Derechos Humanos no se investiguen, persigan o sancionen (p. 54).

\footnotetext{
${ }^{3}$ Nota: esta definición es extendida ya que no solo abarca lo referente al poder público, sino que va más allá de las actividades gubernamentales de cualquier nivel, porque en realidad no se trata de un problema propio o exclusivo de las instituciones gubernamentales ya que también envuelve las relaciones entre los particulares que, de forma independiente o conjunta con aquellas, amplifican en todos los sectores los efectos del fenómeno de la corrupción.
} 


\section{d. Servidores públicos.}

A partir de 1982 se cambió el concepto tradicional de "funcionario público" por el de "servidor público" para deslindar las responsabilidades administrativas (Fraga, 2005, p.130), y de esa manera delimitar la naturaleza del servicio que implica el desempeño de cualquier empleo cargo o comisión, al servicio del Estado o Federación en cualquier nivel, quedando de esa manera establecidas y limitadas las responsabilidades y obligaciones en el servicio público en cualquier ámbito, así lo establece el artículo 108, párrafo primero de la (CPEUM, 1917), misma que los señala de manera enunciativa pero no limitativa, todo esto en relación con los artículos 109, 110, 11 y 113 del mismo documento supremo.

\section{e. Ética de los servidores públicos.}

La Comisión Nacional de Derechos Humanos (CNDH) (2019) refiere "La ética del servidor público orienta el comportamiento al que debe sujetarse en su quehacer cotidiano, para prevenir conflictos y delimitar su actuación en las funciones o actividades que involucren riesgos de posibles actos de corrupción” (p. 2). Por su parte, Verónica Fernández (2014) en su libro La Ética en la Administración Pública: Los Códigos de Conducta. El Caso Español, cita a Oscar Diego, quien refiere que: "La ética pública señala principios y valores deseables para ser aplicados en la conducta del hombre que desempeña una función pública" (p. 32). Mientras que Hernández, et. al. (2017) sostienen que la ética en los servidores públicos comprende el conjunto de principios, valores y normas de conducta que estos deben mostrar en el desempeño de sus funciones, los cuales por lo general suelen estar reflejados dentro de un código de ética a fin de orientar su comportamiento (p. 127). Por su parte, Patricio Orellana (2007) nos dice que "la ética pública (...) se refiere al uso del poder político de quienes administran el Estado" (p. 2). Por tanto, este último autor (2007) continúa señalando: "entendemos la ética pública como los principios y comportamiento de los políticos, los funcionarios públicos y los ciudadanos en relación con la forma en que manejan los recursos que la sociedad reúne para cumplir los fines sociales del Estado” (p. 2).

\section{e. Códigos éticos.}

En el Acuerdo por el que se emite el Código de Ética de las personas Servidoras Públicas del Gobierno Federal, publicado el 5 de febrero de 2019, en el artículo 4, fracción II, se establece que un Código de Ética es el:

Instrumento deontológico, al que refiere el artículo 16 de la Ley General de Responsabilidades Administrativas, que establece los parámetros generales de valoración y actuación respecto al comportamiento al que aspira una persona servidora pública, en el ejercicio de su empleo, cargo o comisión, a fin de promover un gobierno transparente, íntegro y cercano a la ciudadanía.

Siguiendo a Eber Betanzos (2017), un Código de Ética aporta una serie de ideas y conceptos que ayudan a entender la diversidad de criterios morales y a partir de estos 
conceptos es posible cimentar una ética más acorde con los valores, principios y costumbres de una determinada comunidad política, traducido en la capacidad de la persona servidora pública para crear, pensar y desarrollar actitudes más acordes con su visión en la impartición y administración de justicia, siempre en respeto de los Derechos Humanos (p. 30). Asimismo, los Códigos de Ética o de Conducta de la Administración Pública en México se constituyen como un instrumento traducido en la capacidad para crear y motivar su criterio para incidir en una serie de normas, que lejos de limitar o coartar su libertad, le dan los elementos para desarrollar su creatividad acorde con las funciones y niveles a desempeñar dentro de la Función Pública. Además, dichos Códigos contienen principios que no son limitativos única y exclusivamente para el ámbito laboral, sino que traspasan las fronteras de la costumbre, de la cultura y hasta familiares, por lo que el cumplimiento de dicho Código no debe hacerse por temor o por castigo, sino por propio bienestar, conveniencia y por respeto al deber, así como a las funciones encomendadas. Por su parte, Oscar Diego (2005) nos dice: "Los códigos de ética son un instrumento fundamental en el impulso de la ética pública al ser parte del marco normativo que regula la actuación de los miembros que operan dentro de las instituciones públicas.” (p.138):

El código de conducta en el servicio público es una declaración en la que se definen las actitudes esperadas por parte de los servidores públicos señalando además principios éticos aplicables en su ámbito laboral. Abarca también un compendio de valores y antivalores, así como una lista de responsabilidades y obligaciones jurídicas. Ya sean de carácter legal o administrativo, los códigos desempeñan un papel orientador y de control al establecer las restricciones de comportamiento. (p. 138)

\section{PRINCIPIOS CONSTITUCIONALES}

A continuación, vamos a referirnos a algunos de los principios y valores constitucionales como: legalidad, honradez, lealtad, imparcialidad, eficiencia, bien común, integridad, justicia, transparencia, rendición de cuentas, entorno cultural y ecológico, generosidad, igualdad, respeto y liderazgo, que todo servidor público debe observar en el desempeño de su empleo, cargo, comisión o función, por lo que resulta importante la CPEUM y el Código de Ética de los Servidores Públicos del Gobierno Federal (CESPGF, 2015), y también la obra publicada por el Comité de ética y de prevención de conflictos de interés del Instituto Nacional de Lenguas Indígenas (2017).

\section{a. Legalidad.}

Este principio está consignado en los artículos 14,16,109 Fracción III, de la CPEUM y en el (CESPGF, 2015), al que alude igualmente el citado Comité de ética (2017) que a la letra dice: "Los servidores públicos hacen sólo aquello que la norma expresamente les confiere y en todo momento someten su actuación a las facultades que las leyes, reglamentos y demás disposiciones jurídicas atribuyen a su empleo, cargo o comisión...” (p.13). 


\section{b. Honradez.}

Este principio se localiza en el artículo 21, 102, A, Fracción VI, de la CPEUM, expresamente en el (CESPGF, 2015), al que alude el citado Comité de ética (2017), que establece:

Los servidores públicos se conducen con rectitud sin utilizar su empleo, cargo o comisión para obtener o pretender obtener algún beneficio, provecho o ventaja personal o a favor de terceros, ni buscan o aceptan compensaciones, prestaciones, dádivas, obsequios o regalos de cualquier persona u organización.... (p.13).

\section{c. Lealtad.}

Este principio se localiza en el artículo 109, fracción III, de la CPEUM, claramente en el (CESPGF, 2015), al que alude el citado Comité de ética (2017):

Los servidores públicos corresponden a la confianza que el Estado les ha conferido; tienen una vocación absoluta de servicio a la sociedad, y satisfacen el interés superior de las necesidades colectivas por encima de intereses particulares, personales o ajenos al interés general y bienestar de la población (p. 13).

\section{d. Imparcialidad}

A su vez, este principio se observa en el artículo 109, fracción III, de la CPEUM, especialmente en el (CESPGF, 2015), al que alude el citado Comité de ética (2017), que dice: "Los servidores públicos dan a los ciudadanos y a la población en general el mismo trato, no conceden privilegios o preferencias a organizaciones o personas...” (p.13).

\section{e. Eficiencia.}

Por su parte, este principio se observa en el artículo 109, fracción III, de la CPEUM, en el (CESPGF, 2015), e igualmente, a este se refiere el citado Comité de ética (2017), que dice: "Los servidores públicos actúan conforme a una cultura de servicio orientada al logro de resultados, procurando en todo momento un mejor desempeño de sus funciones a fin de alcanzar las metas institucionales según sus responsabilidades..." (pp.13-14).

\section{f. Integridad.}

Este principio lo encontramos en los artículos 21 y 29 de la CPEUM, en el (CESPGF, 2015), e igualmente a este se refiere el citado Comité de ética (2017):

Los servidores públicos actúan siempre de manera congruente con los principios que se deben observar en el desempeño de un empleo, cargo, comisión o función, convencidos en el compromiso de ajustar su conducta para que impere en su desempeño una ética que respon- 
da al interés público... (p. 15).

\section{g. Transparencia.}

Este principio lo encontramos en el artículo 134 de la CPEUM, en el (CESPGF, 2015), e igualmente a este se refiere el precitado Comité de ética (2017): "El servidor público debe permitir y garantizar el acceso a la información gubernamental, sin más límite que el que imponga el interés público y los derechos de privacidad de los particulares establecidos por la ley...” (pp. 15-16).

\section{Rendición de cuentas.}

Este principio lo encontramos en los artículos 6, Fracción VIII y 113, Fracción II de la CPEUM, en el (CESPGF, 2015), e igualmente, a este se refiere el ante citado Comité de ética (2017): "Para el servidor público rendir cuentas significa asumir plenamente ante la sociedad, la responsabilidad de desempeñar sus funciones en forma adecuada y sujetarse a la evaluación de la propia sociedad...” (p.16).

\section{m. Entorno cultural y ecológico.}

Este principio lo encontramos en los artículos 27 de la CPEUM, en el (CESPGF, 2015), e igualmente a este se refiere el ante citado Comité de ética (2017):

Al realizar sus actividades, el servidor público debe evitar la afectación de nuestro patrimonio cultural y del ecosistema donde vivimos, asumiendo una férrea voluntad de respeto, defensa y preservación de la cultura y del medio ambiente de nuestro país, que se refleje en sus decisiones y actos... (p.15).

\section{ñ. Igualdad y no discriminación.}

Este principio lo encontramos en los artículo $2^{\circ}$ Inciso B, 30 párrafo IV, 60, inciso B, Fracción V, 17 párrafo III, 20, inciso A, Fracción V, 115, Fracción II, inciso a), de la CPEUM, en el (CESPGF, 2015), e igualmente a este se refiere el ante citado Comité de ética (2017): "El servidor público debe prestar los servicios que se le han encomendado a todos los miembros de la sociedad que tengan derecho a recibirlos, sin importar su sexo, edad, raza, credo, religión o preferencia política...” (pp. 14-15).

\section{n. Respeto a los derechos humanos.}

Este principio lo encontramos en los artículos $1^{\circ}$ párrafo III, $2^{\circ}$ inciso $\mathrm{B}, 3^{\circ}$ párrafo IV, 60, inciso B, Fracción V, 17 párrafo III, 20, inciso A, Fracción V, 115, Fracción II, inciso a), de la CPEUM, en el (CESPGF, 2015), e igualmente a este se refiere el ante citado Comité de ética (2017): "Los servidores públicos respetan los derechos humanos, y en el ámbito de sus competencias y atribuciones, los garantizan, promueven y protegen de conformidad con los Principios de: Universalidad (...) Interdependencia (...) Indivisibilidad (...) Progresividad...” (p. 14). 
En síntesis, el servidor público debe dar a las personas un trato digno, cortés, cordial y tolerante; además, está obligado a reconocer y conducirse con respeto en todo momento a los derechos humanos y libertades reconocidos en la citada CPEUM. Inclusive, el artículo 109, fracción II de esa Constitución, contempla la responsabilidad penal por la posible comisión de delitos, cuando establece: "La comisión de delitos por parte de cualquier servidor público o particulares que incurran en hechos de corrupción, será sancionada en los términos de la legislación penal aplicable.”

\section{CÓDIGOS DE ÉTICA DE LOS SERVIDORES PÚBLICOS FEDERALES}

A continuación, se abordarán los dos primeros códigos de ética de los servidores públicos federales en México, correspondientes a los años 2002 y 2012 y, posteriormente, los de 2015 y 2019.

\section{a. Códigos de Ética de 2002 y 2012.}

La Ley federal de responsabilidades administrativas de los servidores públicos (LFRASP) publicada en el DOF el día 13 de marzo de 2002 establecía en su artículo 49 la obligación de la Secretaría de la Contraloría y Desarrollo Administrativo (SECODAM), de emitir un código de ética que contendría lo principios y valores de la actuación de los servidores públicos; asimismo, en el artículo $8^{\circ}$ transitorio establecía que dicho código debería emitirse en un plazo no mayor de ciento veinte días hábiles. Este es el origen del primer código de ética de los servidores de la administración pública federal publicado en el DOF el 31 de julio del 2002 (CESAPF-2002). Además, las bases jurídicas de este ordenamiento las encontramos también en el Plan Nacional de Desarrollo 2001-2006 (PND, 2001-2006), que establecía la prevención de las prácticas de corrupción e impunidad y también impulsar la mejora de la calidad en la gestión pública con el propósito de asegurar a la ciudadanía una certidumbre en cuanto a las actividades de las acciones de gobierno y la calidad de sus servicios, garantizando la profesionalización y honestidad de los servidores públicos. También se sustentaba en el Programa Nacional de Combate a la Corrupción y Fomento a la Transparencia y el Desarrollo Administrativo 2001-2006.

Cabe señalar que el segundo Código de Ética de los Servidores Públicos de la Administración Pública Federal publicado en el DOF el 06 de marzo de 2012 (CESAPF), es una transcripción del primer código publicado en 2002. Por tanto, ambos códigos están basados en los mismos principios contemplados en el artículo 113, título IV de la CPEUM. Sus objetivos eran garantizar el cumplimiento de dichos principios, la consolidación de una administración ética, eficaz, transparente y responsable, que rinda cuentas, combata y castigue la arbitrariedad, la corrupción y la impunidad. El Plan Nacional de Desarrollo 2007-2012 (PND, 2007-2012), fue la base del antedicho código de ética de 2012. Lo mismo sucedió con el Programa Nacional de Rendición de Cuentas, Transparencia y Combate a la Corrupción 2008-2012 (PNRCTYCC, 2008-2012), que establecía como una de las estrategias para contribuir al desarrollo de una cultura de 
apego a la legalidad, de ética y responsabilidad pública, la de "Consolidar una cultura de ética e integridad entre los servidores públicos de la Administración Pública Federal”, como ya se señaló, ambos códigos contenían los mismos principios y hacían hincapié en el combate a la corrupción e impunidad.

\section{b. Códigos de Ética de 2015 y 2019.}

Con la publicación del Código de ética (CESPGF-2015), se intenta restructurar las bases ético-normativas que regían los códigos de ética de 2002 y 2012, teniendo como principal finalidad redirigir el enfoque que asegure el buen actuar en el comportamiento ético de los servidores públicos. Todo esto a través de "Reglas de Integridad" para el ejercicio de la función pública, y los "Lineamientos Generales" para propiciar la integridad de los servidores públicos y para implementar acciones permanentes que favorezcan su comportamiento ético. Dicho código regirá —así se decía—, la conducta de los servidores públicos al servicio de las dependencias y entidades de la administración pública federal.

\section{Tabla 1: Cuadro comparativo de los códigos de ética de los servidores públicos del gobierno federal del agosto de 2015 y el 05 de febrero de 2019}

\begin{tabular}{|c|c|c|}
\hline & Código de ética de 2015 & Código de ética de 2019 \\
\hline $\begin{array}{l}\text { Fundamento } \\
\text { Constitucional }\end{array}$ & $\begin{array}{l}\text { A partir de la reforma Constitucional del } \\
27 \text { de Mayo de 2015, en donde se reforman, } \\
\text { adicionan y derogan diversas disposi- } \\
\text { ciones de la CPEUM, en materia de } \\
\text { combate a la corrupción, el fundamento } \\
\text { constitucional de los principios referentes } \\
\text { al ejercicio de la función pública, que se } \\
\text { encontraban establecidos en su artículo } \\
\text { 113, pasarían en su totalidad al artículo 109, } \\
\text { fracción III, de la misma, estableciendo en } \\
\text { este último artículo lo referente a los } \\
\text { servidores públicos y particulares que } \\
\text { incurran en responsabilidad frente al } \\
\text { Estado, dejando al primer artículo mencio- } \\
\text { nado todo lo referente al Sistema Nacional } \\
\text { Anticorrupción. }\end{array}$ & $\begin{array}{l}\text { El artículo } 109 \text { fracción III, de la CPEUM, } \\
\text { establece: } \\
\text { Se aplicarán sanciones administrativas a } \\
\text { los servidores públicos por los actos u } \\
\text { omisiones que afecten la legalidad, honra- } \\
\text { dez, lealtad, imparcialidad y eficiencia } \\
\text { que deban observar en el desempeño de } \\
\text { sus empleos, cargos o comisiones. Dichas } \\
\text { sanciones consistirán en amonestación, } \\
\text { suspensión, destitución e inhabilitación, } \\
\text { así como en sanciones económicas, y } \\
\text { deberán establecerse de acuerdo con los } \\
\text { beneficios económicos que, en su caso, } \\
\text { haya obtenido el responsable y con los } \\
\text { daños y perjuicios patrimoniales } \\
\text { causados por los actos u omisiones. La ley } \\
\text { establecerá los procedimientos para la } \\
\text { investigación y sanción de dichos actos u } \\
\text { omisiones. } \\
\text { Artículo 113. El Sistema Nacional Anticor- } \\
\text { rupción es la instancia de coordinación } \\
\text { entre las autoridades de todos los órdenes } \\
\text { de gobierno competentes en la prevención, } \\
\text { detección y sanción de responsabilidades } \\
\text { administrativas y hechos de corrupción, } \\
\text { así como en la fiscalización y control de } \\
\text { recursos públicos. }\end{array}$ \\
\hline $\begin{array}{l}\text { Principios } \\
\text { constitucionales }\end{array}$ & $\begin{array}{l}\text { Legalidad, honradez, lealtad, imparciali- } \\
\text { dad, eficiencia. }\end{array}$ & $\begin{array}{l}\text { Legalidad, honradez, lealtad, imparciali- } \\
\text { dad, eficiencia. }\end{array}$ \\
\hline
\end{tabular}




\begin{tabular}{|c|c|c|}
\hline $\begin{array}{l}\text { Valores } \\
\text { expuestos en el } \\
\text { código de ética }\end{array}$ & $\begin{array}{l}\text { Interés público, respeto, respeto a los } \\
\text { derechos humanos, igualdad y no discrim- } \\
\text { inación, equidad de género, entorno } \\
\text { cultural y ecológico, integridad, } \\
\text { cooperación, liderazgo, transparencia y } \\
\text { rendición de cuentas. }\end{array}$ & $\begin{array}{l}\text { Legalidad, objetividad, profesionalismo, } \\
\text { honradez, lealtad, imparcialidad, integri- } \\
\text { dad, rendición de cuentas, eficacia y } \\
\text { eficiencia. }\end{array}$ \\
\hline Objetivo & $\begin{array}{l}\text { Regir la conducta de los servidores } \\
\text { públicos al servicio de las dependencias y } \\
\text { entidades de la Administración Pública } \\
\text { Federal. }\end{array}$ & $\begin{array}{l}\text { Art. } 7^{\circ} \text { Establecer las bases y políticas para } \\
\text { la promoción, fomento y difusión de la } \\
\text { cultura de integridad en el servicio } \\
\text { público; establecer las acciones permanen- } \\
\text { tes que aseguren la integridad y el compor- } \\
\text { tamiento ético de las y los servidores } \\
\text { públicos, y crear las bases mínimas para } \\
\text { que todo organo del Estado mexicano } \\
\text { establezca políticas eficaces de ética } \\
\text { pública y responsabilidad en el servicio } \\
\text { público. }\end{array}$ \\
\hline Propósito & $\begin{array}{l}\text { Aplicar sanciones administrativas a los } \\
\text { servidores públicos por los actos u } \\
\text { omisiones que afecten la legalidad, } \\
\text { honradez, lealtad, imparcialidad y eficien- } \\
\text { cia que deban observar en el desempeño de } \\
\text { sus empleos, cargos o comisiones. Asimis- } \\
\text { mo, y considerando las funciones que les } \\
\text { correspondan, establecerán acciones para } \\
\text { delimitar las conductas de sus servidores } \\
\text { públicos en situaciones específicas, previo } \\
\text { diagnóstico que al efecto realicen y } \\
\text { conforme a los lineamientos que emita } \\
\text { esta Secretaría. }\end{array}$ & $\begin{array}{l}\text { Que las personas servidoras públicas } \\
\text { deberán observar en el desempeño de sus } \\
\text { empleos, cargos o comisiones, los referidos } \\
\text { principios éticos. }\end{array}$ \\
\hline $\begin{array}{l}\text { Contenido } \\
\text { General }\end{array}$ & $\begin{array}{l}\text { Establecer las reglas de integridad para el } \\
\text { ejercicio de la función pública para } \\
\text { propiciar la buena conducta de los } \\
\text { servidores públicos e implementar } \\
\text { acciones permanentes que favorezcan su } \\
\text { comportamiento ético, a través de los } \\
\text { comités de ética y de prevención de conflic- } \\
\text { tos de interés. }\end{array}$ & $\begin{array}{l}\text { Establecer un conjunto de principios, } \\
\text { valores y reglas de integridad que orienten, } \\
\text { en un marco de aspiración a la excelencia, } \\
\text { el desempeño de las funciones y la toma de } \\
\text { decisiones de las personas servidoras } \\
\text { públicas, asumiéndolos como líderes en la } \\
\text { construcción de la nueva ética pública, y } \\
\text { constituir el eje, a partir del cual, las } \\
\text { dependencias, entidades y empresas } \\
\text { productivas del Estado, elaboren sus } \\
\text { respectivos códigos de conducta en los que } \\
\text { se consideren riesgos éticos específicos, en } \\
\text { atención a su misión, visión y atribu- } \\
\text { ciones. }\end{array}$ \\
\hline
\end{tabular}

Fuente: elaboración propia con información tomada de los códigos de ética de los servidores públicos del gobierno federal publicados en el DOF el 20 de agosto de 2015 y el 05 de febrero de 2019.

Ahora, la SECODAM era sustituida por la Secretaría de la Función Pública (SFP), quien asumió el compromiso de emitir el citado CESPGF-2015, conforme a las reformas que había tenido la Ley Orgánica de la Administración Pública Federal (LOAPF), publicada en el DOF el 29 desde diciembre de 1976. Mientras tanto, la LFRASP continuaba el compromiso en sus artículos 7, 48 y 49 de refrendar la responsabilidad de los servidores públicos para salvaguardar los principios constitucionales supra mencionados. Mientras que el nuevo Plan Nacional de Desarrollo 2013-2018 
(PND, 2013-2018), también asumía el compromiso de continuar con:

...el combate a la corrupción y el fomento de una mayor rendición de cuentas, entendiendo que la corrupción no era un fenómeno aislado sino la consecuencia de distintas formas de transgresión al Estado de Derecho, por lo que no puede combatirse de manera eficaz persiguiendo solamente a los individuos que han cometido faltas, sino construyendo garantías y creando políticas integrales que permitan disminuir esas conductas (CESPGF-2015, 1).

Ahí mismo se agregaba:

Que el Estado promueve acciones para fortalecer la transparencia, la rendición de cuentas, la legalidad y el combate a la corrupción; sin embargo, los verdaderos cambios se gestan a partir de que los servidores públicos de manera individual y en su conjunto, asumen una verdadera cultura ética y de servicio a la sociedad, convencidos de la dignidad e importancia de su tarea... (CESPGF-2015, 1).

Como se puede observar en la tabla 1, el fundamento constitucional cambia del artículo 113 Título IV, al 109 fracción III, mientras que los principios constitucionales se mantienen intocados. Sin embargo, los principios éticos que se habían mantenido igual en los CESAPF-2002 y CESAPF-2012, ahora bajo los códigos CESPGF-2015 y CEPSPGF-2019, tuvieron algunos cambios, pudiera decirse que profundos, pues de uno a otro solo se mantuvieron los valores de integridad y rendición de cuentas, todos los demás son diferentes. Efectivamente, el citado CEPSPGF-2019 establecía los nuevos principios que deberían observar los servidores públicos a que se refiere la Tabla 1 y a los cuales nos hemos referido, igualmente, en el apartado de principios constitucionales. Con esta nueva ley se dice que se vuelve a poner como prioritario:

...la construcción de una nueva ética pública, la recuperación de la confianza de la sociedad en sus autoridades y el compromiso con la excelencia por parte de las personas servidoras públicas, son prioridades del Gobierno Federal, a fin de lograr la transformación de México hacia un país próspero y ordenado, basado en relaciones sociales justas, democráticas, respetuosas y fraternas (CEPSPGF-2019, 1).

Como se desprende de la Tabla 1, se alude al sistema nacional anticorrupción, por lo que, en el artículo $5^{\circ}$ de la Ley General del Sistema Nacional Anticorrupción (LGSNA) y 7 de la Ley General de Responsabilidades Administrativas (LGRA), publicada en 2016, se hace hincapié en los mencionados principios constitucionales y valores éticos que abordamos en el apartado III de este mismo trabajo. También se puede observar que, al igual que con el tercer CESPGF-2015, con el último código CEPSPGF-2019, también surgen nuevos valores que vienen a reforzar el trabajo de los servidores públicos; aunque, cabe señalar que los principios de rendición de cuentas y transparencia datan de los precitados CESAPF-2002 y CESAPF-2012. Mientras que los 
principios constitucionales supra mencionados contemplados en el artículo 109, Fracción III, se mantienen incólumes, de lo que se desprende que, en todas esas codificaciones sobre el tema de la conducta ética de los servidores públicos federales, existe toda una fundamentación sustantiva y adjetiva tendiente, cada vez más, a fortalecer de manera lógica y explícita esa conducta. Además, se reitera el discurso de que la nueva ley es por el bienestar general de la población y del bien común. (Thiebaut, 1992, pp. 48-49) o, como también lo refiere Montesquieu en su obra El Espíritu de las Leyes, la finalidad de la nueva sociedad es la felicidad de todos (Montesquieu, 2013, XX), es decir, aquí regresamos al discurso de los padres de la filosofía liberal, cuando en la Declaración Francesa de los Derechos del Hombre y del Ciudadano de 1789 (Jellinek 2000) se dice, “...con el fin de que las reclamaciones de los ciudadanos, fundadas en adelante sobre los principios simples e indiscutibles, repercutan siempre en el mantenimiento de la Constitución y de la felicidad de todos...” (p. 172). Pero, también, George Nakhnikian (1993) se refiere a dos aspectos: "decir la verdad como un deber incondicional" y "la felicidad es el bien máximo", como principios objetivos de la ética teórica (p. 9), esto es, en el tema de la felicidad, bien común y el bienestar general, como en muchos otros, la doctrina del liberalismo, antes, y ahora conocido como neoliberalismo-globalización, van de la mano de la propia ética, desde sus orígenes. En el caso que nos ocupa, se puede afirmar que, los cuatro códigos de ética supra mencionados están impregnados de esta filosofía, pero también se puede afirmar que la esencia de estos ordenamientos está en consonancia con el pensamiento de Stone y Nakhnikian, puesto que dichos ordenamientos contienen los ideales a los que se deben adecuar la conducta de los servidores públicos. Sin embargo - como veremos más adelante, infra apartado VI, al revisar los resultados de los estudios realizados por la ONGs-, la teoría se vuelve a confrontar con la realidad.

Por otra parte, cabe señalar que entre ya señalados principios constitucionales y los valores contenidos en los códigos de ética para los servidores públicos federales, existe plena identidad, no solamente respecto a los medios, sino también respecto a los fines que persiguen ambos; aunque, como se hizo notar en el párrafo anterior, hay un abismo entre la teoría y la realidad. También podemos decir que tanto los principios constitucionales como los valores contenidos en los citados códigos, todos son derechos humanos amparados por la CPEUM, sobre todo a partir de la reforma constitucional publicada el 10 de junio de 2011.

\title{
IV. REFORMAS CONSTITUCIONALES EN MATERIA PENAL Y DERECHOS HUMANOS DE 2008 Y 2011
}

\begin{abstract}
a) La reforma en materia penal publicada en el DOF el 18 de junio de 2008, constituyó un nuevo paradigma pues dio paso a la sustitución del modelo penal inquisitivo por el modelo penal acusatorio (Consejo de la Judicatura Federal, 2011; Gómez, 2016; Elizalde, 2018). Todos estos cambios amparados en la teoría garantista de Ferrajoli (2004).

b) La reforma constitucional en derechos humanos publicada en México en el
\end{abstract}


DOF el 10 de junio de 2011, constituyó un nuevo paradigma que dio pauta a un verdadero control difuso de constitucionalidad y convencionalidad, que hasta antes de esa fecha no existía; con dicha reforma surgieron, además, nuevos principios constitucionales como el principio pro persona, interpretación conforme, universalidad, interdependencia, indivisibilidad, progresividad. Una consecuencia de estos cambios fue la necesidad de crear nuevos códigos de ética para los funcionarios públicos federales, como son CESPGF-2015 y CEPSPGF-2019, mismos que se tuvieron que adaptar a los nuevos principios constitucionales y convencionales. De tal manera que, a partir de aquí seguimos viendo una simbiosis entre los principios constitucionales y los valores que encierran los referidos códigos de ética, pues dichos ordenamientos coinciden, como ya se señaló antes, en la prioridad de encauzar la conducta de los servidores públicos para el bienestar y el bien común de la sociedad (Comisión Nacional de Derechos Humanos e Instituto Nacional de Estudios Históricos de la República Mexicana, 2015; Lara, 2015, pp. 65-78).

Por otra parte, si bien es cierto que antes de la precitada reforma constitucional, el juicio de amparo solo podía promoverse para solicitar la protección de los derechos fundamentales establecidos en la parte dogmática de la Constitución, después de esa reforma también es factible de invocarse la parte orgánica de la Constitución como parámetro de control de regularidad constitucional en el juicio de amparo, en términos de la siguiente Tesis Aislada: $1^{\mathrm{a}}$. CCCX/2018 (10a .), 2018732. Gaceta del Seminario Judicial de la Federación, Décima Época, Primera Sala, Libro 61, diciembre de 2018, Tomo I, pág. 355, con el rubro de "Parte orgánica de la constitución. Es apta para invocarse como parámetro de control de regularidad en el juicio de amparo.", donde se establece, entre otros aspectos que:

...esta Suprema Corte reitera en esta Décima Época del Semanario Judicial de la Federación que las personas pueden acudir al juicio de amparo para alegar violaciones a la parte orgánica de la Constitución, siempre y cuando se encuentren cumplidos los requisitos atinentes al grado de afectación exigible conforme a la fracción I del artículo 103 constitucional para poder acceder al juicio de amparo...

\section{ORGANISMOS NO GUBERNAMENTALES INTERNACIONALES}

Como señalamos al inicio de este trabajo, la ONU en el año de 2003 elaboró el Diagnóstico sobre la situación de los Derechos Humanos en México, (véase supra pp. 2-3), donde se pudo observar que ningún ámbito de la administración pública escapó al tema de la corrupción e impunidad. Pero, al lado de este ejercicio también están los trabajos de investigación que realizaron con idénticas metodologías haciendo hincapié en la aplicación de cuestionarios y encuestas, algunas ONGs, tal es el caso de HRW, que en su informe anual 2019 sobre México, aparte de las enormes violaciones a los derechos humanos, hace hincapié en la corrupción institucional del Estado mexicano. Por su 
parte, en el Latinobarómetro, México aparece en el $4^{\circ}$ lugar como país más corrupto, solo por debajo de Colombia, Perú y Brasil. La corrupción ocupa el problema más importante de México como país, con el 14\% de la percepción (2018, p. 58). Mientras que TI en su Índice de Percepción de la Corrupción 2019, dice que México se encuentra en el lugar 28.

Por si esto no fuera suficiente, la reforma penal de gran calado que fue anunciada y presentada el miércoles 15 de enero de 2020 por el gobierno federal y el Dr. Alejandro Gertz Manero, Fiscal General de República Mexicana ante el Senado de la República, es un botón más de muestra sobre lo profundo que está el tema de la corrupción en el Sistema de Justicia Penal y Sistema de Procuración de Justicia, cuando uno de los principales objetivos de la reforma constitucional en México, publicada en el DOF el 18 de junio de 2008 en materia de justicia penal, que dio origen a los juicios orales, se supone que era para solucionar en gran medida el problema de la corrupción e impunidad (Gómez, 2016). El propio Gertz Manero sostenía a principios del año 2020 que "El Sistema de Justicia Penal Acusatorio no sirve y debe ser desmantelado" (Foro Jurídico, 2020, p. 153). Mientras que otro informe de 29 de enero de 2019 de TI, se destaca que México durante el año 2018 era el país más corrupto entre los países de la Organización para la Cooperación y el Desarrollo Económicos (OCDE).

Por su parte, la organización internacional WJP, en su índice de Estado de Derecho 2018, muestra que corrupción, seguridad y justicia son los aspectos que requieren atención urgente (2018). Esta misma organización concluía en su primer informe 2018 que México estaba reprobado en el Estado de Derecho. La corrupción y la falta de investigaciones sólidas ante los delitos son focos rojos que debilitan el Estado de derecho en el país. Esta misma organización en su segundo informe 2019 señaló que (por segundo año consecutivo) México se ubicó en la posición 99 de 126 países evaluados y en la posición 26 de 30 países en la región de América Latina y el Caribe. (WJP, 2019).

México se Estanca en Combate a la Corrupción, así lo afirmó “...el pasado 8 de junio la Consultora América Society/Council of the Américas (AS/COA) en conjunto con Control Riks, quienes publicaron en Nueva York la segunda edición del Îndice de Capacidad para Combatir la Corrupción (CCC 2020), que evalúa la capacidad de los países latinoamericanos para descubrir, castigar y detener la corrupción” (Foro Jurídico, 2020, p. 62):

No obstante, el discurso político del combate a la corrupción y la bandera de la austeridad en las Instituciones del Gobierno Federal, en el CCC 2020 nuestro país ocupa el $8^{\circ}$ lugar de Latinoamérica en su capacidad de descubrir, castigar y detener la corrupción con una puntuación de 4.55 de 10 puntos. Es decir, a pesar del discurso oficial de $\mathrm{AMLO}^{4}$ de estar terminando con la corrupción y ser un tema prioritario en su agenda, el índice CCC 2020 muestra que en la práctica no ha cambiado mucho este fenómeno negativo en México (Foro Jurídico, 2020, p. 62).

\footnotetext{
"Con las siglas "AMLO" se identifica popularmente al actual Presidente Constitucional de los Estados Unidos Mexicanos, Andrés Manuel López Obrador.
} 
Por su parte, México Evalúa refiere en sus Hallazgos 2018 que "En 2018 se observa un rezago a nivel nacional en la consolidación del sistema de justicia penal, ya que ninguna de las entidades federativas logró alcanzar el estándar mínimo” (2019, p. 110), y agrega:

La finalidad del sistema de justicia penal en un Estado democrático de derecho es proveer respuestas satisfactorias a los conflictos derivados de la comisión de un delito (...). Cuando el propósito del sistema de justicia no es cumplido, surge la impunidad, que se traduce en "el principal indicador del fracaso de la justicia.

El índice de impunidad, de acuerdo con los Hallazgos 2018, anda en $96.1 \%$ a nivel nacional (2019, p. 114). Por eso, "a once años del inicio del modelo del Sistema de Justicia Penal Acusatorio, se plantea, entre otras posibilidades, asumir el "fracaso (...) del modelo y dar inicio a un proceso de contrarreforma” (2019, p. 119). En esta confrontación entre la ética pública de los servidores públicos federales en México y los estudios realizados por las mencionadas ONGs, en especial sobre el tema de la corrupción e impunidad, se hace presente Victoria Camps quien en su obra Historia de la ética refiere que el proyecto de la ilustración ha sido un auténtico fracaso (1988). Por ello, parafraseando su pensamiento y con base en los resultados antes mencionados, tal vez se puede afirmar que los principios y valores éticos plasmados en los referidos códigos de ética de 2002, 2012, 2015 y 2019, en México haya sido también un fracaso, toda vez que chocan con la realidad. Como quiera que sea, y tomando en cuenta los resultados que arrojan los estudios de las referidas ONGs, podemos decir, al lado de George Nakhnikian que la jurisprudencia ética es solamente un conjunto de ideales a los que se debe adecuar el orden jurídico (1993). Legislativamente se adecuaron los mencionados ordenamientos jurídico-éticos y también se atendió, en consecuencia, la ética normativa, pero las conductas éticas de los servidores públicos no se ajustaron a esos ideales. Con los mencionados resultados se contradijo los referidos principios y valores éticos (ideales) normativos que se fueron plasmando en cada uno de los citados códigos de ética en la forma prescrita por la propia CPEUM, por lo que dichas normas son constitucional y legalmente válidas. No obstante, se propone seguir apostando a la ética y, en especial, a la ética teórica y a la ética normativa en el caso de los servidores públicos federales para que México pueda salir del abismo donde se encuentra.

En este contexto, cabe destacar que el jueves 12 de noviembre de 2020, el pleno de la Suprema Corte de Justicia de la Nación (SCJN) resolvió por unanimidad de los ministros la inconstitucionalidad de la inhabilitación perpetua o "muerte civil" a los funcionarios públicos y particulares acusados de corrupción. También, declaró inválidas algunas partes normativas del Código Penal del estado de Jalisco y de la Ley de Compras Gubernamentales, Enajenaciones y Contrataciones de Servicios, de la misma entidad (Vela, 2020). Esos ordenamientos fueron reformados mediante decretos 27256/LXII/19; 27265/XII/19, vigentes desde el 11 de mayo de 2019, mismos que fueron impugnados por la Fiscalía General de la República y por la Comisión Nacional de los Derechos Humanos. La propuesta del ministro Pérez Dayán fue aprobada por el pleno de la SCJN, quien resolvió que las sanciones penales y administrativas previstas 
en dichos preceptos son inusitadas y trascendentales y, por tanto, prohibidas por el artículo 22 de la Constitución, pues se consideró que esos ordenamientos, en su parte conducente, resultan incongruentes con la finalidad punitiva prevista en el artículo 18 de la CPEUM. Igualmente, se expuso que con dichas disposiciones se viola tanto el principio de proporcionalidad como el derecho a la reinserción social (Vela, 2020).

\section{REFLEXIONES FINALES}

Parafraseando a Hermann Heller, cuando señala que hay un vínculo indisoluble entre el Derecho Constitucional, la Teoría del Estado y la Ciencia Política, lo mismo podemos decir de la ética, los derechos humanos y el derecho penal; igual podemos señalar con este filósofo del derecho, cuando se refiere a lo teórico y lo práctico de la Teoría del Estado y la Ciencia Política, pues para comprender la ética, los derechos humanos y el derecho penal, también es indispensable abordarlas desde sus enfoques teórico y práctico, o sea, hay que confrontarlas con la realidad. Asimismo, hay un vínculo muy estrecho entre la ética, los derechos humanos y el derecho penal, sustentados en la teoría y en la práctica y, sobre todo, en las teorías de Jeorge Nakhnikian, Hermann Heller y Ferrajoli. Pero, además, las tres están relacionadas con el Derecho Constitucional, a partir, no solamente de la parte dogmática de la CPEUM, sino, igual, en su parte dogmática y sus artículos 20,108, 109, 111 y 113 que, entre otras cuestiones, abarcan la responsabilidad penal por las conductas constitutivas de delito. Por consiguiente, se puede decir que se logró el propósito que nos fijamos en un principio, pues se analizaron comparativamente los cuatro códigos de ética para los servidores públicos federales que han estado vigentes en México, en los años de 2002, 2012, 2015 y 2019, lo que nos permitió conocer, no solamente la forma en que han evolucionado los principios constitucionales, los derechos humanos y los valores éticos que les dieron origen, sino también sus semejanzas y diferencias; asimismo, se identificaron los nuevos valores éticos que se incorporaron durante esa transición. Se destacó también la relación de éstas con el derecho penal, tomando en cuenta que el artículo 109, fracción II de la CPEUM, contempla la posibilidad de sancionar penalmente a los servidores públicos que incurran en responsabilidad frente al Estado.

Asimismo, se pudo observar que existe una simbiosis entre los principios constitucionales y los valores contenidos en los códigos de ética de los servidores públicos federales, pues se puede decir que la mayoría de ellos son considerados derechos humanos, tomando en consideración que son tales no solamente los que contiene la parte dogmática de la CPEUM, sino que en términos de la precitada Tesis Aislada: $1^{\mathrm{a}}$. CCCX/2018 (10ª), 2018732, la parte orgánica de la Constitución también es apta para invocarse como parámetro de control de regularidad en el juicio de amparo. Esto desde luego teniendo presente el principio de definitividad, en los casos en que jurídicamente proceda. No obstante, se señaló que ha habido un gran avance en materia legislativa en el ámbito de la ética pública con los cuatro códigos de referencia y de que estos históricamente se han ido adecuando a las reformas constitucionales con los estudios realizados por algunas ONGs, como fue el caso de HRW, TI, Latinobarómetro, WJP, México Evalúa y otras organizaciones, nos percatamos que hay un 
gran abismo entre la teoría y la realidad, pues los resultados que esos organismos arrojan concluyen que el Estado Mexicano sigue ocupando los primeros lugares en corrupción e impunidad y la explicación a todo esto está en la conducta antiética de los servidores públicos. Asimismo, se considera que a pesar de la gran difusión que las instituciones del Estado han dado a los referidos Códigos de Ética entre los servidores públicos federales, este conocimiento de ninguna manera se ve reflejado en la conducta ética de dichos servidores públicos, por tanto, todos esos avances no han logrado evitar que el Estado Mexicano sea reconocido como uno de los más corruptos, ya que, en palabras de TI, México ha fracasado totalmente en las políticas anticorrupción.

\section{REFERENCIAS}

1. Bastida, et. al. (2004). Teoría General de los Derechos Fundamentales en la Constitución española de 1978. Tecnos, Madrid.

2. Betanzos, E. (2017). “Notas en torno a la ética en el Servicio Público Mexicano. Ética y Administración Pública. Elementos para la formación de una conducta íntegra en los servidores públicos." Revista de Administración Pública, 144, Volumen (LII), número 3, 15-32. Recuperado de http://www.inap.mx/portal/images/pdf/rap144.pdf Fecha de consulta: 14 de noviembre de 2020.

\section{Camps, V. (1988). Historia de la ética. Barcelona, España: Crítica.}

4. Carbonell, M. (2004). Los Derechos Fundamentales en México, México: IINJ-CNDH.

5. Comisión Nacional de Derechos Humanos, (2019). Ética del Servidor Público. Publicación con Número de autorización: CE/MD/17/19, México: CNDH.

6. Comisión Nacional de Derechos Humanos, Instituto Nacional de Estudios Históricos de la República Mexicana (2015). Derechos Humanos en el artículo $1^{\circ}$ Constitucional: obligaciones, principios y tratados. México: CNDH, SEP e INEHRM.

7. Comité de Ética y de prevención de conflictos de interés, (2017). Principios que todo servidor público debe observar en el desempeño de su empleo, cargo, comisión o función. México: Instituto Nacional de Lenguas Indígenas, pp. 1-60. Recuperado de https://site.inali.gob.mx/pdf/Principios_servidores_publicos.pdf Fecha de consulta: 12 de noviembre de 2020 .

8. Consejo de la Judicatura Federal y Poder Judicial de la Federación, (2011). El nuevo sistema de justicia penal acusatorio desde la perspectiva constitucional. México: Poder judicial de la Federación, Suprema Corte de Justicia de la Nación, Consejo de la Judicatura Federal y Secretaría Técnica.

9. De Zan, J. (2004). La ética, los derechos y la justicia. Montevideo, Uruguay: Mastergraf. 
10. Diario Oficial de la Federación (05 de febrero de 2019). Código de Ética de las personas Servidoras Públicas del Gobierno Federal, (2019). Recuperado de https://dof. gob.mx/nota_detalle.php?codigo $=5549577 \&$ fecha $=05 / 02 / 2019$ Fecha de consulta: 25 de noviembre de 2020.

11. Diario Oficial de la Federación (13 de marzo de 2002) Ley Federal de Responsabilidades Administrativas. Recuperado de http://www.diputados.gob.mx/LeyesBiblio/abroga.htm Fecha de consulta: 20 de noviembre de 2020.

12. Diario Oficial de la Federación (18 de julio de 2016) Ley General de Responsabilidades Administrativas, (2016). Recuperado de http://www.diputados.gob.mx/LeyesBiblio/index.htm Fecha de consulta: 23 de noviembre de 2020.

13. Diario Oficial de la Federación (18 de julio de 2016) Ley General del Sistema Nacional Anticorrupción. Recuperado de http://www.diputados.gob.mx/LeyesBiblio/index.htm Fecha de consulta: 23 de noviembre de 2020.

14. Diario Oficial de la Federación (20 de agosto de 2015). Código de Ética de los Servidores Públicos del Gobierno Federal. Recuperado de https://www.gob.mx/sfp/documentos/codigo-de-etica-de-los-servidores-publicos-del-gobierno-federal-94426 Fecha de consulta: 20 de noviembre de 2020.

15. Diario Oficial de la Federación (22 de abril de 2002) Programa Nacional de Combate a la Corrupción y Fomento a la Transparencia y el Desarrollo Administrativo (2001-2006). Recuperado de http://www.dof.gob.mx/nota_detalle.php?codigo=734662 \&fecha=22/04/2002 Fecha de consulta: 23 de noviembre de 2020.

16. Diario Oficial de la Federación (29 de diciembre de 1976) Ley Orgánica de la Administración Pública Federal. Recuperada de http://www.diputados.gob.mx/LeyesBiblio/index.htm Fecha de consulta: 23 de noviembre de 2020.

17. Diario Oficial de la Federación (30 de mayo de 2001) Plan Nacional de Desarrollo, (2001-2006). Recuperado de https://www.gob.mx/cms/uploads/attachment/file/22317/PLAN1.pdf Fecha de consulta: 17 de noviembre de 2020.

18. Diario Oficial de la Federación (31 de diciembre de 1982) Ley Federal de Responsabilidades de los Servidores Públicos. Recuperado de http://www.diputados.gob.mx/LeyesBiblio/index.htm Fecha de consulta: 23 de noviembre de 2020.

19. Diario Oficial de la Federación (31 de julio de 2002). Código de Ética de los Servidores Públicos de la Administración Pública Federal, México, Disponible en: https://dof.gob.mx/nota_detalle.php?codigo=727307\&fecha=31/07/2002 Fecha de consulta: 20 de noviembre de 2020.

20. Diario Oficial de la Federación (5 de febrero de 1917) Constitución Política de los Estados Unidos Mexicanos, (CPEUM). Recuperado de http://www.diputados.gob.mx/LeyesBiblio/index.htm Fecha de consulta: 20 de noviembre de 2020. 
21. Diario Oficial de la Federación ( 5 de febrero del 2019). Acuerdo por el que se emite el Código de Ética de las personas servidoras públicas del Gobierno Federal, México. Disponible en: https://dof.gob.mx/nota_detalle.php?codigo=5549577\&fecha=05/02/ 2019 Fecha de consulta: 14 de noviembre de 2020.

22. Diario Oficial de la Federación (6 de marzo de 2012). Código de Ética de los Servidores Públicos de la Administración Pública Federal. Recuperado de http://dof.gob.mx/nota_detalle.php?codigo=5236535\&fecha=06/03/2012 Fecha de consulta: 14 de noviembre de 2020.

23. Diccionario de la Lengua Española, (2014). $23^{\mathrm{a}}$ ed. Disponible en https://dle.rae.es/ Fecha de consulta: 5 de noviembre de 2020.

24. Diego O. (2005). "Los Códigos Éticos en el Marco de las Administraciones Públicas Contemporáneas. Valores para un Buen Gobierno”. Revista de las Cortes Generales, número 65, España.

25. Diego, O. (2009). Ética para corruptos. Una forma de prevenir la corrupción en los gobiernos y administraciones públicas. España: Desclée de Brower.

26. Elizalde, R. (2018). "La impunidad en México: signo de debilidad del Estado de derecho en el modelo neoliberal”, en Diego y Rodríguez (Coords.) La lógica neoliberal y su impacto en el Estado mexicano. Un enfoque multidisciplinario. Chiapas: Universidad Autónoma de Chiapas, UNACH, pp. 83-112.

27. Fernández, V. (2014). La Ética en la Administración Pública: Los Códigos de Conducta. El Caso Español. España: Universidad de Cádiz.

28. Fernández. S. F. (1992). "La dimensión axiológica del Derecho Constitucional", Cuadernos de la Cátedra Fadrique Furio Ceriol, N. 15, Universidad de Santiago, Valencia, pp. 15-34.

29. Ferrajoli, L. (2004). Derechos y Garantías. La ley del más débil. $4^{a}$ ed., Roma: Trotta.

30. Foro Jurídico (2 de julio 2020). Combate a la Corrupción en Latinoamérica. En México se estanca en Combate a la Corrupción. México. Recuperado de https://forojuridico.mx/combate-a-la-corrupcion-en-latinoamerica-en- mexico-se-estanca-en-combate-a-la-corrupcion/ Fecha de consulta: 23 de noviembre de 2020.

\section{Fraga, Gabino (2005). Derecho Administrativo. México: Porrúa.}

32. Gertz, M.A. (30 de enero 2020). "El Sistema de Justicia Penal Acusatorio no sirve, Gertz Manero defiende su propuesta.” Revista en línea Foro Jurídico, 07, 17, p. 156. México. Recuperado de https://forojuridico.mx/el-sistema-de-justicia-penal-acusatorio-no-sirve-gertz-manero-defiende-su-propuesta/ Fecha de consulta: 23 de noviembre de 2020. 
33. Gómez, G. A. (Coord.), (2016). Reforma Penal 2008-2016. El Sistema Penal Acusatorio en México. México: INACIPE.

34. Hernández, P., et. al. (2017). Importancia de la Formación Ética de los Servidores Públicos y de la Aplicación de la Ley para Garantizar un Desempeño Íntegro. (Tesis de Licenciatura). Facultad de Ciencias Administrativas y Sociales de la Universidad Veracruzana, Veracruz, México.

35. Human Righs Watch, (2019). Informe mundial. México: eventos del 2018.

36. Jellinek, J. (2000). La Declaración de los Derechos del Hombre y del Ciudadano. Traducción y estudio preliminar de Adolfo Posada, México: UNAM.

37. Lara Ponte, Rodolfo H. (2015). "La reforma de derechos humanos de 2011. Hacia el Estado constitucional.” En Carbonell, Miguel, Fix Fierro, Héctor, González Pérez, Luis Raúl y Valadés, Diego (Coords.). Estado constitucional, derechos humanos, justicia y vida universitaria. Tomo V, vol. 2, México: UNAM-IIJ.

38. Latinobarómetro, (2018). Informe anual. Corporación Latinobarómetro. Banco de datos en línea. Recuperado de: http://www.latinobarometro.org/lat.jsp

39. Le Clercq, et. al, (2016). Midiendo la impunidad en América Latina: retos conceptuales y metodológicos. Revista de Ciencias Sociales de la Facultad Latinoamericana de Ciencias Sociales, núm. 55, pp. 69-91, sede Académica de Ecuador. Recuperado de https://www.redalyc.org/pdf/509/50945652004.pdf Fecha de consulta: 29 de noviembre de 2020.

40. Le Clercq, J. A. (2018). El problema de la impunidad generalizada: explicando el desempeño de México en el Îndice Global de Impunidad. Revista Espacios Públicos, México, nùm. 51, pp. 51-73. Recuperado de http://politicas.uaemex.mx/espaciospublicos/eppdfs/N51-3.pdf Fecha de consulta: 27 de noviembre de 2020.

41. México Evalúa (2019). Hallazgos 2018: Seguimiento y evaluación del sistema de justicia penal en México. México: México Evalúa, Centro de análisis de políticas públicas.

42. Monereo, P. J.L. (2005). “La teoría de Estudios Político- Jurídica de Hermann Heller”, Revista de Estudios Políticos (Nueva Época), Madrid, pp. 371-400.

43. Montesquieu (2013). Del Espíritu de las Leyes, versión castellana de Nicolás Estébanez, 19ª ed., Porrúa, México, Colección “Sepan cuantos...”, núm. 191, México.

44. Nakhnikian, G. (1993). El Derecho y las Teorías Éticas Contemporáneas. Trad. De Eugenio Bulygin y Genaro R. Carrió, $2^{\mathrm{a}}$ ed. México: Fontamara.

45. Nogueira, A. H. (2003). Teoría y dogmática de los derechos fundamentales. México: UNAM. 
46. Ochoa, R. (2017). Corrupción. Significado y Estrategias Internacionales y Nacionales para su Prevención y Persecución. México: IIJ-UNAM.

47. Oficina del Alto Comisionado de las Naciones Unidas para los Derechos Humanos, (2003). Diagnóstico sobre la situación de los derechos humanos en México. México.

48. Orellana, P. (2007). “Crisis de la ética pública en Chile”. Polis (en línea), núm.16, pp. 1-26. Recuperado de URL: http://journals.openedition.org/polis/4817 Fecha de consulta: 27 de noviembre de 2020.

49. Prado, J. (2016). La moral y la ética: Piedra angular en la enseñanza del derecho. México: Universidad Autónoma de Nuevo León.

50. Rowland, M. (2003). “Visión contemporánea de la corrupción”. En: Abstencionismo y participación electoral, Cuaderno Capel núm. 49-Instituto Interamericano de Derechos Humanos, México: IIJ-UNAM, Ecuador: Fundación Esquel. pp. 1-8. Recuperado de https://archivos.juridicas.unam.mx/www/bjv/libros/11/5005/18.pdf Fecha de consulta: 2 de diciembre de 2020.

51. Sandoval, I. (2016). Enfoque de la corrupción estructural: poder, impunidad y voz ciudadana. Universidad Nacional Autónoma de México-Instituto de Investigaciones Sociales. Revista Mexicana de Sociología, vol. 78, núm. 1, México. Recuperado de http://www.scielo.org.mx/scielo.php?script=sci_arttext\&pid=S0188-2503201600010 0119 Fecha de consulta: 5 de diciembre de 2020.

52. Tesis: $1^{\mathrm{a}}$. CCCX/2018 (10 ${ }^{\mathrm{a}}$.). Seminario Judicial de la Federación y su Gaceta, Décima Época, Primera Sala, Libro 61, diciembre de 2018, Tomo I, pág. 355, con el rubro de "Parte orgánica de la constitución. Es apta para invocarse como parámetro de control de regularidad en el juicio de amparo.”

53. Thiebaut, C. (1992). Neoaristotelismos contemporáneos. En: Campos, et. al. (eds.) Concepciones de la ética, Madrid, España: Trotta y Consejo Superior de Investigaciones Científicas.

54. Transparencia Internacional, (2019). Índice de Percepción de la Corrupción 2019. Recuperado de http://www.rendircuentas.org/noticia/indice-percepcion-la-corrupcion -2019/ Fecha de consulta: 2 de diciembre de 2020.

55. Transparencia Internacional, (2019). México, el más corrupto entre los países de la OCDE. Recuperado de https://www.infobae.com/america/mexico/2019/01/29/mexicoel-mas-corrupto-entre-los-paises-de-la-ocde-transparencia-internacional/ Fecha de consulta: 12 de diciembre de 2020.

56. Vela, D. S. (12/II/20). SCJN Declara inconstitucional la "muerte civil" por corrupción. El Financiero. Recuperado de https://www.elfinanciero.com.mx/nacional/scin-declara-inconstitucional-la-muerte-civil-por-corrupcion Fecha de consulta 14 de nov. de 2020. 
57. Vilchez, Y. (2012). Ética y Moral. Una mirada desde la gerencia pública. Revista Formación Gerencial, núm. 2, México, pp. 232-247. https://dialnet.unirioja.es/servlet/articulo?codigo=4192166 Fecha de consulta: 5 de diciembre de 2020 .

58. World Justice Proyect, (2018). Indice de Estado de Derecho junio 5 2017-2019. Recuperado de https://worldjusticeproject.org/sites/default/files/documents/2017-18\%20ROL I\%20Spanish\%20Edition.pdf Fecha de consulta: 5 de diciembre de 2020.

59. World Justice Proyect, (2019). Índice de Estado de Derecho en el mundo 2019. Recuperado de https://worldjusticeproject.mx/wp-content/uploads/2019/08/GROLI-Spanish. pdf?utm_source=google\&utm_medium=pdf\&utm_campaign=rolindex_spaFecha de consulta: 5 de diciembre de 2020 .

60. World Justice Proyect, (octubre 8, 2018). Índice de Estado de Derecho en México 2018. Recuperado de https://worldjusticeproject.mx/indice-de-estado-de-derecho-en- mexico-2018/ Fecha de consulta: 5 de diciembre de 2020. 